\title{
XÂY DỰNG Bộ TIÊU CHÍ ĐÁNH GIÁ NHANH NĂNG LỰC ĐỔI MỚI SÁNG TAOO CHO KHU VƯC DOANH NGHIÊP NHỎ VÀ VỪA: KINH NGHIỆM QUỐC TẾ VÀ GỢI Ý CHO VIẸTT NAM ${ }^{1}$
}

\author{
Đinh Tuấn Minh², Cao Thu Anh, Đặng Thu Giang \\ Viện Chiến lược và Chính sách khoa học và công nghệ
}

\section{Tóm tắt:}

Mục tiêu của nghiên cứu này là xây dựng bộ tiêu chí đánh giá nhanh năng lục đổi mới sáng tạo (NLĐMST) cho khu vục DNNVV Việt Nam. Để đạt muc tiêu này, trước hết nhóm tác giả tiến hành nghiên cứu tổng quan tài liệu về khái niệm (ĐMST) và NLĐMST của doanh nghiệp và cách thức xây dựng các tiêu chí đánh giá NLĐMST của doanh nghiệp. Bộ tiêu chí này được sử dụng để đánh giá thử NLĐMST tại 121 DNNVV của ba ngành dệt may, chế biến thực phẩm và thiết bị điện-điện tử của Việt Nam. Dựa trên quá trình cũng nhu kết quả đánh giá thử, nghiên cứu này cho rằng các co quan nhà nước và doanh nghiệp có thể sử dụng bảng khảo sát và hệ thống tính điểm chúng tôi đề xuất để đánh giá nhanh NLĐMST của doanh nghiệp và của ngành. Chúng tôi cũng khuyến nghị, Bảng khảo sát không nhất thiết phải đưa vào các câu hỏi định lượng. Việc tính điểm cũng không cần thiết phải gán trọng số khác nhau cho các cấu phần. Ngoài ra, chúng tôi cho rằng nên có một số điều chỉnh nhỏ về ngôn tù̀ trong Bảng khảo sát hiện tại (chủ yếu dùng cho các doanh nghiệp trong lĩnh vục chế biến) khi áp dụng cho các doanh nghiệp cung cấp dịch vu.

Tù khóa: Năng lục đổi mới sáng tạo; Bộ tiêu chí đánh giá; Doanh nghiệp nhỏ và vùa .

Mã số: 19010702

\section{Mở đầu}

Đổi mới sáng tạo từ lâu được coi như là một yếu tố quan trọng giúp doanh nghiệp có thể tồn tại, cạnh tranh và thành công. Hầu hết các nghiên cứu thực nghiệm và điều tra doanh nghiệp cho thấy ĐMST dẫn đến sự ra đời của các sản phẩm, dịch vụ mới có chất lượng tốt hơn và giá thành giảm thấp hơn (Gamal, 2011). Nhưng để tiến hành được các hoạt động ĐMST, doanh nghiệp cần có NLĐMST (Lawson và Samson, 2001).

Việc đo lường NLĐMST của doanh nghiệp là hết sức cần thiết. Trước hết, các kết quả đo lường sẽ giúp doanh nghiệp có cái nhìn rõ ràng hơn về năng

\footnotetext{
${ }^{1}$ Nghiên cứu này là một phần kết quả của Nhiệm vụ khoa học cấp bộ "Nghiên cứu đề xuất định hướng chính sách hỗ trợ nâng cao năng lực đổi mới sáng tạo của doanh nghiệp nhỏ và vừa Việt Nam" thuộc Chương trình: 4198, Mã số: 02-4198-2017 do nhóm tác giả thực hiện.

${ }^{2}$ Liên hệ tác giả: dinhtuanminh.maastricht@gmail.com
} 
lực hiện tại của mình, xác định thế mạnh và các điểm yếu cần phải tập trung đầu tư để tối ưu hóa hiệu quả của hoạt động ĐMST. Các doanh nghiệp cũng sẽ có cơ sở để so sánh năng lực của mình với các doanh nghiệp khác trong cùng ngành hoặc trong so sánh quốc tế để có chiến lược phù hợp. Việc đo lường này cũng sẽ cung cấp các đầu vào quan trọng cho quá trình hoạch định chính sách, có thể giúp trả lời các câu hỏi như đâu là động lực để doanh nghiệp ĐMST và các doanh nghiệp này đang gặp những khó khăn gì trong ĐMST, nhằm thiết kế được các chính sách hỗ trợ hay can thiệp hiệu quả nhất để nâng cao NLĐMST của ngành hay của cả khu vực doanh nghiệp nói chung. Các kết quả đo lường NLĐMST sẽ tạo nên bộ cơ sở dữ liệu phong phú phục vụ cho việc nghiên cứu, đồng thời, việc đánh giá và xếp hạng cũng giúp nâng cao nhận thức của các doanh nghiệp và của cộng đồng về tầm quan trọng của ĐMST, các thành tố góp phần tạo nên NLĐMST của doanh nghiệp, cũng như góp phần thúc đẩy văn hóa ĐMST nói chung. Đó là lý do vì sao nhiều nước trên thế giới đã thực hiện các cuộc điều tra định kỳ để đánh giá NLĐMST của các DN nói chung, mà trong đó phần lớn là các DNNVV. Tiêu biểu là Khảo sát ĐMST cộng đồng chung châu Âu, Chỉ số ĐMST NESTA của Anh, 1-InnoCERT của Malaysia, InnoBiz của Hàn Quốc, IMP3rove của Ủy ban châu Âu dành riêng cho các DNNVV,...

Tại Việt Nam, cho đến nay, vẫn chưa có một khảo sát quy mô, thường xuyên nào về NLĐMST cho các doanh nghiệp nói chung và DNNVV nói riêng. Một số khảo sát của Tổng cục Thống kê hoặc Viện Quản lý Kinh tế Trung ương và các đối tác (2013) thường không chuyên sâu vào hoạt động $\mathrm{DMST}^{3}$. Các khảo sát thường chỉ tập trung vào hoạt động đầu tư $\mathrm{R} \& \mathrm{D}$ tại các doanh nghiệp, trong khi theo như kết quả của các khảo sát này, có tới hơn $90 \%$ doanh nghiệp Việt Nam không có hoạt động R\&D nào. Điều này là dễ hiểu vì đa phần các doanh nghiệp Việt Nam có quy mô nhỏ. Hoạt động ĐMST đối với DN quy mô nhỏ phần nhiều là các ĐMST mang tính mới đối với bản thân $\mathrm{DN}$ hoặc với thị trường của $\mathrm{DN}$. Các doanh nghiệp nhỏ thường quan tâm đến ĐMST nhỏ thay vì là các ĐMST lớn, đòi hỏi nhiều chi phí R\&D.

Với những thông tin còn chưa đầy đủ về NLĐMST của doanh nghiệp Việt Nam, chúng tôi nhận thấy cần phải xây dựng một khung khổ và hệ tiêu chí đánh giá mới, chắt lọc từ các phương pháp đã được phát triển và áp dụng

\footnotetext{
${ }^{3}$ Trong năm 2017, Cục Thông tin KH\&CN Quốc gia, đã tiến hành cuộc điều tra thử nghiệm để hoàn thiện công tác thống kê ĐMST trong các doanh nghiệp tại Việt Nam giai đoạn 2014-2016. Theo công bố tại Hội thảo "Kết quả thống kê ĐMST trong doanh nghiệp và đề xuất hoàn thiện công tác thống kê ĐMST ở Việt Nam" ngày 28/8/2018, cuộc điều tra này đã tiến hành khảo sát tại 7.641 doanh nghiệp ngành công nghiệp chế biến, chế tạo, trong đó, có 1.892 doanh nghiệp lớn (chiếm $67,84 \%$ tổng số doanh nghiệp lớn), 820 doanh nghiệp vừa (chiểm $90,01 \%$ ) và 4.929 doanh nghiệp nhỏ (chiến 26,25\%). Tuy nhiên, cho tới thời điểm chúng tôi viết bài báo này, kết quả chính thức của cuộc khảo sát vẫn chưa được công bố. Vì lẽ đó, chúng tôi chưa thể có bình luận hoặc trích dẫn chính thức về cuộc điều tra này.
} 
trên thế giới, để đo lường NLĐMST của các doanh nghiệp của Việt Nam. Riêng với các DNNVV, do số lượng nhiều và liên tục thay đổi, việc xây dựng một bộ tiêu chí để đánh giá nhanh NLĐMST là rất cần thiết để thường xuyên đánh giá, bên cạnh một bộ tiêu chí đầy đủ, chuyên sâu về NLĐMST. Vì vậy, mục đích của bài viết này là nghiên cứu tổng quan về cách thức xây dựng các tiêu chí đánh giá NLĐMST của doanh nghiệp đã được thực hiện ở trong và ngoài nước. Từ đó, đề xuất xây dựng bộ tiêu chí đánh giá nhanh NLĐMST cho khu vực DNNVV. Để đạt được mục tiêu này, trong phần tiếp theo nghiên cứu sẽ phân tích khái niệm ĐMST và NLĐMST của doanh nghiệp. Tiếp đến, nghiên cứu sẽ xem xét các phương pháp và công cụ đo lường ĐMST đã được sử dụng trên thế giới. Cuối cùng nghiên cứu sẽ đề xuất bộ tiêu chí để đánh giá sơ bộ NLĐMST cho các DNNVV của Việt Nam. Bộ tiêu chí này sẽ được sử dụng để đánh giá thử NLĐMST 121 DNNVV tại ba ngành dệt may, chế biến thực phẩm và thiết bị điện-điện tử của Việt Nam.

\section{Tổng quan lý thuyết}

\subsection{Khái niệm đổi mới sáng tạo và năng lục đổi mới sáng tạo}

Theo Schumpeter (1934), ĐMST là một quá trình mà chủ doanh nghiệp đưa các kết hợp mới ra thị trường. Đó có thể là việc đưa ra sản phẩm mới, áp dụng phương pháp sản xuất mới hoặc cách thức bán hàng mới, mở ra một thị trường mới, sử dụng một nguồn cung ứng nguyên liệu đầu vào mới, hay hình thành một cấu trúc thị trường mới.

Các định nghĩa về ĐMST sau này về cơ bản kế thừa các ý tưởng chính ở trên của Schumpeter, nhưng có những điều chỉnh để phù hợp với mục tiêu của từng nghiên cứu cụ thể. Trong các nghiên cứu ứng dụng hiện nay, phiên bản khái niệm về ĐMST được dùng phổ biến nhất là của $\mathrm{OECD/Eurostat}$ (1992, 1997, 2005), được đưa ra trong Cuốn sổ tay Oslo (Oslo Manual) về việc thu thập và diễn giải các dữ liệu đổi mới công nghệ để phục vụ cho mục đích đo lường các hoạt động khoa học, công nghệ và ĐMST. Theo Oslo Manual (2005), ĐMST là quá trình đưa vào áp dụng một sản phẩm hoặc quy trình mới, một phương pháp tiếp thị mới, hoặc một phương pháp tổ chức mới hoặc cải thiện đáng kể trong hoạt động sản xuất, kinh doanh. Các tổ chức này cũng cho rằng, yêu cầu tối thiểu cho việc xác định ĐMST sản phẩm, quy trình, tiếp thị hay tổ chức là hoạt động đó phải là mới hoặc được cải thiện đáng kể. Dựa trên khái niệm này, các tổ chức này phân loại ĐMST tại cấp doanh nghiệp thành 4 loại: (i) ĐMST sản phẩm; (ii) ĐMST quy trình; (iii) ĐMST marketing; và (iv) ĐMST tổ chức.

Năng lực ĐMST của doanh nghiệp được định nghĩa là khả năng tổ chức các nguồn lực để thực hiện được một số hoạt động ĐMST nhất định nào đó 
(Neely et al, 2001), hoặc chi tiết hơn, đó là khả năng biến đổi liên tục tri thức và ý tưởng thành các sản phẩm mới, qui trình sản xuất mới, mở ra các thị trường mới, cách thức tổ chức mới để giúp doanh nghiệp và các thành viên của nó hưởng lợi (Lawson và Samson, 2001).

Để thể hiện được khái niệm trên dưới dạng các hoạt động có thể đo lường được, ở bước cơ bản nhất, chúng ta có thể nhìn NLĐMST như là một tập hợp các yếu tố đầu vào của doanh nghiệp (Romijn và Albaladajo, 2000). Cụ thể, năng lực ĐMST của doanh nghiệp được hình thành từ nhiều nguồn bên trong và bên ngoài doanh nghiệp. Các nguồn bên trong bao gồm: nền tảng kiến thức của người chủ hoặc quản lý doanh nghiệp, các kỹ năng của đội ngũ nhân viên và nỗ lực đầu tư cho $R \& D$. Các nguồn bên ngoài bao gồm tần suất quan hệ với bên ngoài, độ sâu của các mối quan hệ với bên ngoài, mức độ nhận được hỗ trợ từ bên ngoài.

Bước tiếp theo, chúng ta nhìn NLĐMST theo quá trình ĐMST. Có nhiều cách nhìn quá trình ĐMST khác nhau. Theo cách nhìn truyền thống, chúng ta có thể xem quá trình ĐMST bao gồm các công đoạn như phát minh, phát triển, khai thác. Một cách nhìn khác đó là theo chuỗi giá trị ĐMST (innovation value chain) như Hansen and Birkinshaw (2007) đề xuất. Theo các tác giả này, một chuỗi giá trị ĐMST bao gồm ba giai đoạn: tạo ra ý tưởng, chuyển đổi ý tưởng ra kết quả và lan tỏa ý tưởng.

Ở mức độ sâu hơn nữa, chúng ta cần phải cân nhắc đến cả yếu tố bối cảnh hoặc bầu không khí hình thành NLĐMST (Nilsson và cộng Sư, 2012). Bầu không khí ĐMST (innovation climate) là một tập hợp các yếu tố mang tính thể chế như các giá trị chung, mức độ tự do sáng tạo, chế độ động viên khuyến khích, thái độ mạo hiểm,... Bầu không khí sáng tạo được xem như là một phần của NLĐMST vì nó chính là điều kiện để giúp cho ĐMST có khả năng xảy ra cao hơn.

\subsection{Các phương pháp đo luờng năng lục đổi mới sáng tạo của doanh nghiệp}

2.2.1 Các khung khổ lý thuyết về đo lương năng lục đổi mói sáng tạo của doanh nghiệp

Các khung lý thuyết về đo lường NLĐMST hiện đại đều xem xét ĐMST ở rất nhiều chiều và nhiều công đoạn trong quá trình ĐMST, thay vì chỉ đơn thuần đo lường các đầu vào hay đầu ra của ĐMST của doanh nghiệp. Mô hình kim cương, được đề xuất bởi Tidd, Bessant, và Pavit (2005), đo lường năm chiều của NLĐMST, bao gồm chiến lược, quy trình, tổ chức, các mối liên kết và học hỏi. Mô hình kim cương chủ yếu tập trung vào năng lực nội tại của chính doanh nghiệp, mà không tính đến bối cảnh hay môi trường trong đó doanh nghiệp hoạt động. Mô hình này giúp đưa ra những đánh giá 
khá toàn diện về cơ cấu tổ chức và văn hóa của doanh nghiệp trong việc thúc đẩy việc học hỏi và lan tỏa tri thức, trong việc quản lý và vận hành quy trình phát triển sản phẩm, trong việc lập chiến lược, kế hoạch, thực thi các ĐMST và đánh giá ĐMST; và trong việc tạo ra một văn hóa khuyến khích ĐMST trong doanh nghiệp. Tuy nhiên, mô hình này chưa đề cập đến năng lực của doanh nghiệp trong việc thương mại hóa các sản phẩm ĐMST của mình.

Khung khổ của OSLO Manual, được xây dựng bởi OECD và Hội đồng châu Âu (European Commission), cung cấp một phân loại rất rõ ràng các hình thức ĐMST, bao gồm 4 loại (ĐMST sản phẩm/dịch vụ, ĐMST quy trình, ĐMST tổ chức và ĐMST marketing) của doanh nghiệp để đưa ra cách tiếp cận đo lường phù hợp. Phiên bản mới nhất của OSLO Manual đã bổ sung thêm ĐMST ở lĩnh vực dịch vụ, điều mà các phiên bản trước và các cách thức đo lường ĐMST trước đó chưa tính đến. Cách tiếp cận này cho phép đo lường các đầu vào của ĐMST, các mối liên kết và vai trò của việc lan tỏa, tác động của ĐMST (ví dụ tác động đến sản lượng, năng suất, việc làm ở cả cấp quốc gia và trong các ngành cụ thể hoặc khu vực cụ thể), các động lực và trở ngại của hoạt động ĐMST và các yếu tố về phía cầu. Khung khổ này tạo điều kiện cho việc đo lường NLĐMST ở cấp độ quốc gia và cho phép việc đối chiếu so sánh ở cấp độ quốc tế. Các kết quả của phương pháp này rất hữu ích cho việc hoạch định chính sách (từ các thông tin về yếu tố nào thúc đẩy doanh nghiệp ĐMST hay các trở ngại đối với ĐMST mà doanh nghiệp gặp phải).

Mô hình hình phễu, do trường Học viện Công nghệ Massachuset (MIT) phát triển vào năm 2005 , và mô hình chuỗi giá trị ĐMST, được đề xuất bởi Hansen và Birkinshaw (2007), có thể áp dụng hiệu quả hơn đối với các doanh nghiệp có quy trình ĐMST tuyến tính hơn là các quy trình ĐMST phức tạp và với các phản hồi trong quy trình đó. Thông thường, quy trình tuyến tính giúp cho doanh nghiệp có thể quản lý được ĐMST sử dụng một hệ thống giống như "cửa ra vào", sàng lọc ý tưởng nào đủ tốt hoặc không đủ tốt để đưa đến các giai đoạn tiếp theo của quy trình. Điều này có thể giúp đảm bảo an toàn cho tổ chức, nhưng đôi khi lại mất thời gian, và phù hợp với các ĐMST theo kiểu cải biến hơn là các ĐMST có tính chất đột phá. Điểm yếu của các mô hình này là chưa tính đến các yếu tố bên ngoài tác động đến NLĐMST của doanh nghiệp như là thể chế, cơ sở hạ tầng, nhu cầu của thị trường, mức độ cạnh tranh của ngành mà doanh nghiệp đang hoạt động, các chính sách cạnh tranh, sở hữu trí tuệ và các chính sách hỗ trợ ĐMST của chính phủ.

2.2.2 Thực tiễn đo luờng NLĐMST doanh nghiệp tại một số quốc gia trên thế giới

> Khảo sát ĐMST của Cộng đồng chung châu Âu 
Khảo sát ĐMST của Cộng đồng chung châu Âu (Community Innovation Survey - CIS) được thiết kế dựa trên khung khổ của OSLO Manual, được thực hiện lần đầu tiên vào năm 1992. Kể từ năm 2008, khảo sát này được thực hiện định kỳ hai năm một lần ở các nước thành viên Liên minh châu Âu (EU). Điều tra này được thực hiện ở cấp độ doanh nghiệp. CIS định nghĩa một doanh nghiệp là doanh nghiệp ĐMST nếu như doanh nghiệp đó có ít nhất một sản phẩm hoặc quy trình mới đối với chính doanh nghiệp đó (Arundel, 2007). Việc thu thập số liệu CIS là tự nguyện cho các nước thành viên, do vậy, số lượng các nước tham gia vào mỗi vòng điều tra có thể khác nhau. Các số liệu này có thể được truy cập từ trang web thống kê của Liên minh châu Âu (Eurostat, 2017).

Cuộc điều tra này khảo sát nhiều khía cạnh khác nhau của hoạt động ĐMST như là các sản phẩm/dịch vụ mới hoặc được cải tiến đáng kể; và việc áp dụng các quy trình, logistics hay phương thức phân phối mới hoặc đã được cải thiện. Điều tra cũng cung cấp thông tin về đặc điểm của hoạt động ĐMST ở cấp độ doanh nghiệp, giúp doanh nghiệp có cái nhìn sâu hơn về quy trình ĐMST và tác động của ĐMST đến nền kinh tế. Các câu hỏi của điều tra cung cấp một tập hợp các chỉ số về các nội dung, các hoạt động ĐMST (sản phẩm, quy trình, tổ chức, ...), chi tiêu cho ĐMST, tác động của ĐMST, tài trợ của chính phủ, hợp tác trong ĐMST, các nguồn thông tin cho ĐMST, các yếu tố cản trở và thúc đẩy hoạt động ĐMST, lý do thực hiện ĐMST và các phương pháp bảo hộ quyền sở hữu trí tuệ.

> Chi Số ĐMST NESTA

NESTA là Quỹ tài trợ quốc gia về khoa học và nghệ thuật của Anh với mục đích thúc đẩy năng lực ĐMST của quốc gia này. Quỹ hướng đến hỗ trợ cho các công ty trong giai đoạn mới thành lập, cung cấp cho họ thông tin về các chính sách liên quan đến ĐMST và khuyến khích họ xây dựng văn hóa thúc đẩy ĐMST trong doanh nghiệp. Chỉ số ĐMST của NESTA được xây dựng từ năm 2008, nhằm mục đích xác định một loạt các chỉ số phản ánh năng lực ĐMST ở mỗi ngành và giúp so sánh năng lực ĐMST giữa các ngành nhằm xác định các ưu tiên chính sách và chiến lược.

Bộ chỉ số ĐMST của NESTA được xây dựng dựa trên mô hình chuỗi giá trị ĐMST của Hansen và Birkinshaw (2007), với cách tiếp cận đầu cuối (endto-end) của quá trình ĐMST, từ lúc doanh nghiệp đầu tư cho tri thức cho đến ĐMST và cuối cùng là tạo ra giá trị. Theo cách tiếp cận này, các hoạt động ĐMST được xem như là một quá trình liên tục gồm ba pha với đầu tư cho tri thức, thực hiện ĐMST và tạo giá trị (thương mại hóa các ĐMST).

NESTA xây dựng 16 chỉ số ĐMST cấp doanh nghiệp. Năm (05) trong số các chỉ số này liên quan đến việc tiếp cận tri thức; 6 chỉ số liên quan đến việc tạo ra các ĐMST; 5 chỉ số liên quan đến hoạt động thương mại hóa. Ở 
một số trường hợp, các chỉ số được định nghĩa hoàn toàn mang tính chất đa ngành - tức là cùng một chỉ số có thể áp dụng cho nhiều ngành khác nhau. Một số chỉ số chỉ áp dụng cho từng ngành cụ thể.

\section{$>$ Radar $Đ M S T$}

Radar ĐMST là một công cụ được phát triển bởi Trường Quản lý Kellogg School, gồm các nhà nghiên cứu Mohan Sawhney, Robert $\mathrm{C}$. Wolcott và Inigo Arroniz (Gamal, 2011).

Công cụ này đã được kiểm chứng và được áp dụng cho 40 công ty ở Mỹ. Công cụ này đưa ra một cái nhìn toàn diện về ĐMST và kết quả cho thấy các doanh nghiệp đưa ĐMST vào mô hình kinh doanh có kết quả tốt hơn so với các doanh nghiệp chỉ tập trung vào ĐMST đối với sản phẩm hoặc quy trình.

Radar đưa ra bốn khía cạnh đóng vai trò như là mỏ neo của hoạt động kinh doanh:

- Cung cấp thứ mà công ty tạo ra (Cái gì - WHAT);

- Các khách hàng mà công ty phục vụ (Ai - WHO);

- Quá trình sử dụng (như thế nào - HOW);

- Điểm hiện diện mà hãng sử dụng để đưa các sản phẩm của mình ra thị trường (Ở đâu - WHERE).

Trải rộng ở cả 4 khía cạnh này, các doanh nghiệp có thể đổi mới hoạt động của họ nhiều hơn so với việc chỉ tập trung vào ĐMST công nghệ hoặc sản phẩm: một doanh nghiệp thực tế có thể tiến hành ĐMST ở 12 chiều cạnh khác nhau. Radar ĐMST sẽ giúp mở rộng phạm vi ĐMST của doanh nghiệp, và cho thấy rằng "ĐMST là tạo ra các giá trị mới, chứ không phải là tạo ra các sản phẩm mới".

\section{$>$ 1-InnoCeRT}

1-InnoCERT là một chương trình hướng đến thúc đẩy hoạt động ĐMST của doanh nghiệp ở Malaysia. Chương trình này đánh giá bốn khía cạnh cơ bản: Khả năng ĐMST, Khả năng thương mại hóa, Khả năng quản lý ĐMST và Kết quả ĐMST. Chương trình cấp chứng nhận cho các doanh nghiệp ĐMST thông qua một giấy chứng nhận quy trình tuân thủ và khả năng doanh nghiệp có thể tuân thủ theo những tiêu chuẩn về ĐMST nhất định. Việc cấp giây chứng nhận được thực hiện dựa trên hệ thống tự đánh giá ĐMST trực tuyến và được chứng thực thông qua hoạt động kiểm toán thực tế tại hiện trường. 1-InnoCERT mở rộng với tất cả các doanh nghiệp (SMEs và các doanh nghiệp quy mô lớn) trong 8 ngành bao gồm: chế tạo, dịch vụ, công nghệ sinh học, thiết kế, công nghệ thông tin và truyền thông/phần 
mềm, nông nghiệp, môi trường, công nghệ xanh (năng lượng tái tạo...) và xây dựng.

\section{> InnoBiz}

Hàn Quốc thực hiện hệ thống chứng nhận ĐMST công nghệ từ năm 2001 để hỗ trợ các DNNVV thực hiện ĐMST. Việc đánh giá dựa trên "Sổ tay Oslo" do OECD xây dựng. Quá trình đánh giá gồm 2 giai đoạn: tự đánh giá trực tuyến và đánh giá tại hiện trường.

Tụ đánh giá trục tuyến (đánh giá sơ bộ ban đầu)

Việc đánh giá này bao gồm 4 lĩnh vực (năng lực ĐMST công nghệ, năng lực thương mại hóa công nghệ, năng lực quản lý ĐMST công nghệ, và thành tựu ĐMST) với khoảng 60 câu hỏi.

Điểm cao nhất là 1.000 điểm. Và doanh nghiệp cần đạt từ 650 điểm trở lên để được lựa chọn đánh giá tại giai đoạn tiếp.

Đánh giá tại hiện trường của quỹ đảm bảo công nghệ

- Đánh giá hệ thống ĐMST công nghệ (điểm cao nhất: 1.000 điểm): từ 700 điểm trở lên để được lọt vào vòng Đánh giá trình độ công nghệ.

Áp dụng các chỉ số đánh giá tại thời điểm tự đánh giá (đánh giá sơ bộ ban đầu) và đánh giá bởi các chuyên gia đặc biệt của Quỹ Công nghệ Kibo.

- Đánh giá trình độ công nghệ chuyên sâu (hệ thống điểm 10): hạng B hoặc cao hơn để được quỹ cấp tài trợ

Việc đánh giá trình độ công chuyên sâu sẽ gồm có 4 nội dung (năng lực kĩ thuật của người quản lý, khả năng đứng vững của công nghệ, khả năng marketing, mức độ lâu dài và có lợi nhuận của ngành kinh doanh) và khoảng 44 hạng mục.

Đánh giá trình độ công nghệ chuyên sâu gồm 10 cấp độ: $\mathrm{AAA}, \mathrm{AA}, \mathrm{A}$, $\mathrm{BBB}, \mathrm{BB}, \mathrm{B}, \mathrm{CCC}, \mathrm{CC}, \mathrm{C}$, và $\mathrm{D}$.

\section{$>$ IMP3rove - Europe Innova}

Dự án IMP3rove được Ủy ban châu Âu lập ra từ năm 2006 nhằm mục đích thúc đẩy ĐMST của các DNNVV ở châu Âu với tác động bền vững. Dự án này bắt đầu với việc phân tích các cách thức quản lý ĐMST và các công cụ tự đánh giá tốt nhất ở châu Âu. Việc đánh giá được thực hiện một cách hệ thống ở tất cả các chiều cạnh của "Ngôi nhà ĐMST của A.T Kearrney". Nó đo lường các yếu tố chính cần thiết cho sự thành công của ĐMST, bao gồm chiến lược ĐMST, tổ chức và văn hóa, và quản lý vòng đời (bao gồm quản lý ý tưởng, phát triển sản phẩm, quá trình ra mắt sản phẩm, và cải tiến liên tục). 
IMP3rove áp dụng cách tiếp cận toàn diện để đánh giá quản lý ĐMST như là một nhân tố chính thúc đẩy năng lực cạnh tranh. IMP3rove tích hợp việc đánh giá trực tuyến, đưa ra tiêu chuẩn, các dịch vụ tư vấn và cải tiến liên tục quản lý ĐMST của các DNNVV. Bước đầu tiên là thực hiện các đánh giá IMP3rove trực tuyến, với các kết quả được báo cáo chi tiết về kết quả đánh giá của doanh nghiệp ở mỗi chiều của quản lý ĐMST, so sánh với các doanh nghiệp đang có kết quả tốt nhất và so với mức trung bình của các doanh nghiệp trong bộ dữ liệu. Các báo cáo này sẽ được nghiên cứu kĩ lưỡng bởi các nhà tư vấn để chuẩn bị cho các hội thảo tư vấn, nhằm xây dựng một lộ trình cho việc nâng cao hiệu quả quản trị ĐMST của doanh nghiệp. Các lộ trình này phải đưa ra được các mục tiêu và cách thức cụ thể để thực hiện. Việc thực hiện của doanh nghiệp sẽ được theo dõi chặt chẽ, cùng với các đánh giá tác động trong ngắn hạn (ngay sau khi đưa ra dịch vụ tư vấn) và trong dài hạn hơn (sau một năm). Quy trình này sẽ được lặp lại sau một năm nhằm đảm bảo sự cải thiện liên tục trong hệ thống quản trị ĐMST của doanh nghiệp.

\subsubsection{Thảo luận về các phương pháp đo lương năng lực đổi mới sáng tạo}

Bảng 1 dưới đây tổng hợp lại các mô hình và công cụ đo lường NLĐMST của doanh nghiệp, các mối quan tâm chính của mỗi mô hình và những lưu ý cho việc áp dụng các mô hình này. Mô hình kim cương là khung khổ để xây dựng IMP3rove của châu Âu nhằm đánh giá quản lý ĐMST của doanh nghiệp. Bộ chỉ số ĐMST NESTA dựa trên mô hình chuỗi giá trị ĐMST. Các công cụ như 1-InnoCERT, InnoBiz hay CIS của châu Âu dựa trên khung khổ được đưa ra trong OSLO Manual. Mỗi bộ công cụ tập trung vào những yếu tố cốt lõi khác nhau của quá trình ĐMST và có những thế mạnh riêng. Mỗi doanh nghiệp, tổ chức đánh giá, hoặc chương trình quốc gia có thể cân nhắc và tích hợp những cấu phần khác nhau của các mô hình và công cụ đã đề cập để phục vụ cho mục đích của mình.

Bảng 1: So sánh các phương pháp đo lường NLĐMST của doanh nghiệp

\begin{tabular}{|c|c|c|c|c|}
\hline $\begin{array}{l}\text { Mô hình } \\
\text { đo lường }\end{array}$ & $\begin{array}{l}\text { Ví dụ về các } \\
\text { công cụ }\end{array}$ & Trọng tâm & Các chiều kích & Ghi chú \\
\hline $\begin{array}{l}\text { Mô hình } \\
\text { Kim } \\
\text { cương }\end{array}$ & - IMP3rove & $\begin{array}{l}\text { - Quá trình } \\
\text { ĐMST } \\
\text { - Các yếu tố } \\
\text { kích hoạt } \\
\text { - Các mối liên } \\
\text { kết }\end{array}$ & $\begin{array}{l}\text { Chiến lược, quy } \\
\text { trình, tố chức, } \\
\text { các mối liên kêt } \\
\text { và học hỏi. }\end{array}$ & $\begin{array}{l}\text { Đầy đủ khi quá trình } \\
\text { ĐMST còn trong giai } \\
\text { đoạn mới bắt đầu. } \\
\text { Phương pháp này nhấn } \\
\text { mạnh các chiều chính } \\
\text { của quá trình ĐMST } \\
\text { cũng như các yếu tố thể } \\
\text { chế cho phép ĐMST. }\end{array}$ \\
\hline Mô hình & & $\begin{array}{l}\text { - Tập trung } \\
\text { vào ĐMST }\end{array}$ & $\begin{array}{l}\text { Tư duy chiến } \\
\text { lược, quản lý }\end{array}$ & $\begin{array}{l}\text { Mô hình đầy đủ khi có } \\
\text { một quy trình ĐMST }\end{array}$ \\
\hline
\end{tabular}




\begin{tabular}{|c|c|c|c|c|}
\hline $\begin{array}{l}\text { Mô hình } \\
\text { đo lường }\end{array}$ & $\begin{array}{c}\text { Ví dụ về các } \\
\text { công cụ }\end{array}$ & Trọng tâm & Các chiều kích & Ghi chú \\
\hline phễu & & $\begin{array}{l}\text { công nghệ hoặc } \\
\text { sản phẩm } \\
\text { - Quy trình } \\
\text { R\&D như là } \\
\text { hoạt động cốt } \\
\text { lõi }\end{array}$ & $\begin{array}{l}\text { danh mục và } \\
\text { các thước đo, } \\
\text { nghiên cứu, đưa } \\
\text { ý tưởng, tìm } \\
\text { hiểu khách } \\
\text { hàng, đưa ra } \\
\text { mục tiêu, phát } \\
\text { triển ĐMST, } \\
\text { phát triển thị } \\
\text { trường, và bán } \\
\text { hàng. }\end{array}$ & thích hợp trong tổ chức. \\
\hline $\begin{array}{l}\text { Chuỗi } \\
\text { giá trị } \\
\text { ĐMST }\end{array}$ & -NESTA & $\begin{array}{l}\text { - Quản lý ý } \\
\text { tưởng } \\
\text { - Kết quả đầu } \\
\text { ra }\end{array}$ & $\begin{array}{l}\text { Tạo ra ý tưởng, } \\
\text { chuyển hóa ý } \\
\text { tưởng, lan tỏa } \\
\text { Thu thập tri } \\
\text { thức, xây dựng } \\
\text { các ĐMST và } \\
\text { thương mại hóa } \\
\text { các ĐMST. }\end{array}$ & $\begin{array}{l}\text { Nhấn mạnh việc đánh } \\
\text { giá đầu ra của quá trình } \\
\text { ĐMST. }\end{array}$ \\
\hline \multirow[t]{2}{*}{$\begin{array}{l}\text { Hướng } \\
\text { dẫn } \\
\text { OSLO }\end{array}$} & $\begin{array}{l}\text { - InnoCERT } \\
\text { - InnoBiz } \\
\text { - Khảo sát } \\
\text { ĐMST của } \\
\text { Cộng đồng } \\
\text { chung châu } \\
\hat{\text { Âu }} \\
\end{array}$ & $\begin{array}{l}\text { - ĐMST } \\
\text { - Các mối liên } \\
\text { kết } \\
\text { - Đầu ra trong } \\
\text { một thời gian } \\
\text { nào đó }\end{array}$ & $\begin{array}{l}\text { ĐMST, các mối } \\
\text { liên kêt, nhu } \\
\text { cầu, cơ sở hạ } \\
\text { tầng và khung } \\
\text { khồ thể chế, và } \\
\text { chính sách } \\
\text { ĐMST. }\end{array}$ & $\begin{array}{l}\text { Rất hữu ích khi xem xét } \\
\text { ở cấp độ quốc gia; } \\
\text { thuận tiện cho việc so } \\
\text { sánh quốc tế. }\end{array}$ \\
\hline & $\begin{array}{l}\text { - Radar } \\
\text { ĐMST }\end{array}$ & $\begin{array}{l}\text { - Kết quả đầu } \\
\text { ra của ĐMST }\end{array}$ & $\begin{array}{l}\text { Các sản } \\
\text { phẩm/dịch vụ; } \\
\text { khách hàng, } \\
\text { quy trình, } \\
\text { marketing. }\end{array}$ & $\begin{array}{l}\text { Không đảm bảo tính } \\
\text { bền vững của quá trình } \\
\text { ĐMST. }\end{array}$ \\
\hline
\end{tabular}

Nguồn: Tổng hợp bởi nhóm tác giả

\section{Xây dựng bộ tiêu chí đánh giá sơ bộ năng lực đổi mới sáng tạo cho khu vực doanh nghiệp nhỏ và vừa của Việt Nam}

Đã có một số phương pháp đo lường NLĐMST được áp dụng ở Việt Nam như bộ công cụ i2METRIC (Quan Hoang Vuong và cộng sư, 2014) hay bộ tiêu chí khảo sát phục vụ nghiên cứu thực nghiệm của Phùng Xuân Nhạ và Lê Quân (2013). Tuy nhiên, các phương pháp này đều thiếu một khung lý thuyết làm cơ sở cho việc xây dựng các bộ chỉ tiêu đánh giá, do vậy, hệ thống các tiêu chí đưa ra chưa thực sự thuyết phục. Một số công trình nghiên cứu của Viện Chiến lược và Chính sách khoa học và công nghệ cũng đã động chạm đến khía cạnh ĐMST (Nguyê̂n Việt Hòa, 2008; Nguyến 
Việt Hòa, 2011; Trần Ngọc Ca, Nguyễn Võ Hung, 2012); cũng như NLĐMST (Bạch Tân Sinh, 2010) ở cấp độ doanh nghiệp. Nhưng những nghiên cứu này hoặc chỉ mới đề cập đến một số khía cạnh của ĐMST hoặc chưa hệ thống hóa được cơ sở lý thuyết cũng như kinh nghiệm thực tiễn tại các quốc gia cho việc xây dựng các tiêu chí ${ }^{4}$.

Để có cách tiếp cận toàn diện hơn đối với NLĐMST, trước hết chúng tôi áp dụng khung lý thuyết được trình bày trong Oslo Manual 2005 về ĐMST. Theo đó, ĐMST ở đây được hiểu là các hoạt động tạo ra giá trị mới cho doanh nghiệp. Các đối tượng của ĐMST được quan tâm xem xét bao gồm: ĐMST đối với sản phẩm/dịch vụ; ĐMST đối với quy trình; ĐMST đối với hoạt động Marketing; và ĐMST đối với tổ chức.

Tuy nhiên, khung khổ của OSLO Manual 2005 quan tâm nhiều đến đầu ra của ĐMST và các mối liên kết của doanh nghiệp với bên ngoài (thể chế và cơ sở hạ tầng, các doanh nghiệp khác, khu vực đào tạo và hệ thống nghiên cứu công, và nhu cầu của thị trường). Để đánh giá NLĐMST của doanh nghiệp, chúng ta quan tâm cả đến cấp độ vi mô đối với các quy trình ĐMST bên trong doanh nghiệp, từ khâu tìm kiếm tri thức, tạo ra các ĐMST đến khai thác các ĐMST. Tất cả đều phản ánh năng lực ĐMST của doanh nghiệp. Các mô hình lí thuyết khác mà chúng tôi đã đề cập ở trên (Mô hình kim cương, mô hình hình phễu, mô hình chuỗi giá trị ĐMST) đều quan tâm đến nhiều khía cạnh hơn của năng lực ĐMST chứ không chỉ nhấn mạnh vào đầu ra và các mối liên kết của doanh nghiệp với bên ngoài. Tuy thế, mô hình hình phễu phân ra quá chi tiết các quy trình của ĐMST mà đối với các doanh nghiệp quy mô vừa và nhỏ, và các doanh nghiệp chưa có quy trình ĐMST một cách chuyên nghiệp sẽ rất khó có thể phân ra được chi tiết các khâu như vậy. Mô hình chuỗi giá trị ĐMST tỏ ra phù hợp hơn đối với DNNVV khi chỉ phân loại 3 giai đoạn chính: (i) tạo ý tưởng; (ii) chuyển đổi ý tưởng; và (iii) lan tỏa ý tưởng (thương mại hóa ĐMST).

Vì thế, Bộ tiêu chí đánh giá nhanh NLĐMST của DNNVV của Việt Nam được chúng tôi đề xuất xây dựng cần dựa trên cả khung khổ của OSLO Manual 2005 lẫn mô hình chuỗi giá trị ĐMST để phản ánh cả khía cạnh đầu ra, mối quan hệ với bên ngoài, và quy trình bên trong về hoạt động ĐMST tại doanh nghiệp.

\footnotetext{
${ }^{4}$ Chúng tôi cũng lưu ý, theo công bố tại Hội thảo "Kết quả thống kê ĐMST trong doanh nghiệp và đề xuất hoàn thiện công tác thống kê ĐMST ở Việt Nam" ngày 28/8/2018, trong năm 2017, Cục Thông tin Khoa học và Công nghệ Quốc gia đã tiến hành Cuộc điều tra thử nghiệm về ĐMST trong các doanh nghiệp tại Việt Nam giai đoạn 2014-2016. Cuộc điều tra tập trung vào 4 nhóm tiêu chí cơ bản: (i) hoạt động ĐMST của doanh nghiệp; (ii) các điều kiện cơ bản cần thiết cho ĐMST; (iii) tác động tích cực của ĐMST đối với sản xuất kinh doanh của doanh nghiệp và (iv) nguyên nhân cản trở hoạt động ĐMST của doanh nghiệp. Tuy nhiên, như lưu ý ở Chú thích 3 , cho tới thời điểm chúng tôi viết bài báo này, kết quả chính thức của cuộc khảo sát vẫn chưa được công bố. Vì lẽ đó, chúng tôi chưa thể có bình luận hoặc trích dẫn chính thức về cuộc điều tra này.
} 


\subsection{Các cấu phần của NLĐMST}

Tổng hợp từ hai mô hình trên và các ứng dụng cụ thể tại các quốc gia, chúng tôi đưa ra khung khổ về NLĐMST cho DNNVV của Việt Nam bao gồm 4 cấu phần chính.

(1) Năng lực quản lý ĐMST (S), bao gồm:

- Các yếu tố hỗ trợ việc tạo ra ý tưởng (văn hóa của doanh nghiệp khuyến khích việc tạo ra các ý tưởng mới, văn hóa hợp tác chia sẻ kiến thức trong nội bộ doanh nghiệp, văn hóa coi trọng các ý tưởng dến từ bên ngoài, khả năng đưa ra các ý tưởng mới từ nội bộ, khả năng tìm kiếm các ý tưởng mới từ bên ngoài);

- Các yếu tố hỗ trợ việc phát triển ý tưởng (quy trình chọn lọc ý tưởng để phát triển, thái độ với rủi ro, khả năng hoàn thành dự án ĐMST đúng kế hoạch, mức độ hào hứng của đội ngũ quản lý với các dự án ĐMST);

- Các yếu tố hỗ trợ việc lan tỏa ý tưởng (khả năng nhanh chóng đưa ĐMST ra thị trường trước các đối thủ cạnh tranh, khả năng đưa các kết quả ĐMST đến các kênh phân phối).

(2) Năng lực học hỏi và đầu tư cho ĐMST (I), bao gồm:

- Năng lực học hỏi từ bên ngoài (mức độ nhận được tri thức từ các nhà cung ứng, khách hàng, các trường đại học, viện nghiên cứu, từ các cơ quan chính phủ, từ các hiệp hội ngành nghề, từ các triển lãm, hội chợ);

- Năng lực học hỏi nội bộ (mức độ trao đổi tri thức trong nội bộ doanh nghiệp, đào tạo cho nhân viên);

- Đầu tư cho hoạt động R\&D (mức độ đầu tư cho R\&D, mua máy móc, thiết bị mới);

- Nỗ lực ĐMST (thể hiện qua các dự án ĐMST đã triển khai, đã bị dừng hoặc từ bỏ, trì hoãn).

(3) Kết quả ĐMST $(\mathrm{O})$, bao gồm:

- Kết quả ĐMST sản phẩm;

- Kết quả ĐMST quy trình;

- Kết quả ĐMST tổ chức;

- Kết quả ĐMST marketing.

(4) Năng lực thương mại hóa kết quả ĐMST $(M)$, bao gồm:

- Đầu tư cho việc giới thiệu sản phẩm mới ra thị trường (mức độ đầu tư cho việc giới thiệu sản phẩm mới ra thị trường);

- Tác động đến kết quả kinh doanh từ các kết quả ĐMST (tác động đến doanh thu, thị phần, quan hệ với khách hàng, mức độ hài lòng của khách hàng). 


\subsection{Tính điểm năng lục đổi mới sáng tạo}

1. Tính điểm của các nhóm thành phần NLĐMST của DN:

$$
\mathrm{S}=\sum \mathrm{S}(\mathrm{i}) ; \mathrm{I}=\sum \mathrm{I}(\mathrm{i}) ; \mathrm{O}=\sum \mathrm{O}(\mathrm{i}) ; \mathrm{M}=\sum \mathrm{M}(\mathrm{i})
$$

Trong đó: i là thứ tự các tiêu chí trong Bộ tiêu chí;

$\mathrm{S}^{(\mathrm{i})}$ là số điểm của tiêu chí thứ i trong nhóm $\mathrm{S}$;

$\mathrm{I}^{(\mathrm{i})}$ là số điểm của tiêu chí thứ i trong nhóm $\mathrm{I}$;

$\mathrm{O}^{(i)}$ là số điểm của tiêu chí thứ i trong nhóm $\mathrm{O}$;

$\mathrm{M}^{(\mathrm{i})}$ là số điểm của tiêu chí thứ i trong nhóm $\mathrm{M}$.

$>$ 2. Tính tổng số điểm các nhóm thành phần NLĐMST của DN:

$$
\tau=\mathrm{S}+\mathrm{I}+\mathrm{O}+\mathrm{M}
$$

Ví dụ: Sau khi đánh giá một doanh nghiệp có kết quả nhu sau: nhóm năng lực quản lý hoạt động ĐMST được 25 điểm; nhóm đầu tu phát triển năng lục ĐMST được 12 điểm; nhóm kết quả ĐMST được 13 điểm và nhóm tổ chức thuơng mại hóa được 15 điểm. Ta có:

- Tổng số điểm các thành phần ĐMST của DN là:

$$
\tau=25+12+13+15=65 \text { diểm }
$$

Sau khi tính điểm cho mỗi doanh nghiệp, chúng tôi sẽ tổng hợp để đưa ra mức điểm trung bình (hoặc trung vị) của các doanh nghiệp. Để đánh giá NLĐMST của mỗi ngành và đưa ra so sánh, chúng tôi sẽ tiến hành so sánh theo từng khía cạnh của NLĐMST và năng lực tổng hợp. Điểm của mỗi chiều cạnh của một ngành được tính là điểm trung bình (hoặc trung vị) của tất cả các doanh nghiệp trong cùng một ngành. Cộng một cách cơ học điểm của từng chiều cạnh của mỗi ngành, chúng tôi sẽ được điểm chung cho NLĐMST của từng ngành (điểm thấp nhất là 0 , cao nhất là 100 ).

\section{3. Đánh giá thử năng lục đổi mới sáng tạo của một số doanh nghiệp nhỏ và vừa của Việt Nam}

\subsubsection{Kết quả đánh giá thư}

Bộ tiêu chí đánh giá NLĐMST trên đã được chúng tôi dùng để đánh giá cho 121 DNNVV Việt Nam ở 3 ngành công nghiệp: chế biến thực phẩm (42 doanh nghiệp), thiết bị điện-điện tử (21 doanh nghiệp), và dệt may (48 doanh nghiệp). Đây là các doanh nghiệp chúng tôi nhận được phản hồi sau khi gửi Phiếu khảo sát một cách ngẫu nhiên tới 950 doanh nghiệp trong cả nước.

Cần lưu ý rằng, do số lượng phiếu khảo sát thu được tương đối thấp (khoảng $10 \%$ số phiếu gửi đi), dẫn đến sự hạn chế về tính chính xác của kết 
quả phân tích và đánh giá cho điểm, nên kết quả phân tích ở đây chỉ nhằm mục đích hoàn thiện phương pháp cũng như bộ tiêu chí, thay vì rút ra các hàm ý về NLĐMST cho ba ngành trên cũng như toàn bộ khu vực DNNVV của Việt Nam.

Bảng 2 tổng hợp kết quả đánh giá NLĐMST của các doanh nghiệp trong mẫu khảo sát tại mỗi ngành. Điểm số trong mỗi chiều cạnh của NLĐMST tại mỗi ngành được tính bằng cách chia trung bình NLĐMST tại mỗi chiều cạnh của các doanh nghiệp khảo sát trong ngành.

Về tổng thể, NLĐMST của các ngành đều ở mức trung bình. Tuy nhiên, ngành chế biến thực phẩm có NLĐMST cao nhất, đạt 60,92/100 điểm, còn ngành thiết bị điện-điện tử có NLĐMST thấp nhất, đạt 44,75/100 điểm. Về các điểm thành phần, duy nhất chỉ có Năng lực quản lý hoạt động ĐMST trong ngành chế biến thực phẩm đạt mức cao và kết quả ĐMST trong ngành điện-điện tử đạt mức thấp, còn lại đều ở mức trung bình.

Kết quả trên phản ánh phần nào thực tế phát triển của ba ngành này tại Việt Nam trong những năm vừa qua. Các doanh nghiệp nội địa trong ngành chế biến thực phẩm và dệt may đã có nhiều bứt phá, không chỉ đáp ứng tốt nhu cầu tiêu thụ trong nước mà còn đóng góp nhiều cho xuất khẩu. Trong khi đó, ngành thiết bị điện-điện tử đều bị thống lĩnh bởi các doanh nghiệp FDI và hàng nhập khẩu. Các $\mathrm{DNNVV}$ trong ngành này gặp khó khăn trong việc thâm nhập được vào trong chuỗi giá trị toàn cầu của các doanh nghiệp FDI.

So sánh các cấu phần, năng lực quản lý hoạt động ĐMST của các doanh nghiệp trong tất cả các ngành đều tốt hơn các năng lực khác. Cả ba ngành đều đạt mức cao hoặc gần ngưỡng cao. Điều này cho thấy, các DNNVV đều có ý thức và mong muốn ĐMST. Trong khi đó, năng lực tạo ra kết quả ĐMST thấp nhất trong các năng lực. Kết quả này phản ánh việc tạo ra những kết quả ĐMST cụ thể là khó khăn đối với các DNNVV. Một trong những khó khăn mà các doanh nghiệp phản ánh là thiếu vốn đầu tư cho ĐMST.

Bảng 2: So sánh NLĐMST của các doanh nghiệp trong các ngành chế biến thực phẩm, dệt may và thiết bị điện-điện tử

\begin{tabular}{|l|c|c|c|c|l|}
\hline & $\begin{array}{c}\text { Điểm } \\
\text { tối đa }\end{array}$ & $\begin{array}{c}\text { Chế biến } \\
\text { thực phẩm }\end{array}$ & $\begin{array}{c}\text { Dệt } \\
\text { may }\end{array}$ & $\begin{array}{c}\text { Thiết bị } \\
\text { điện-điện tử }\end{array}$ & Khoảng điểm \\
\hline Số DN khảo sát & & 42 & 48 & 21 & \\
\hline $\begin{array}{l}\text { Năng lực quản lý } \\
\text { hoạt động ĐMST }\end{array}$ & 30 & 20,38 & 17,78 & 18,73 & $\begin{array}{l}\text { Cao: }>20 \\
\text { Trung bình: } 10-20 \\
\text { Thấp: }<10\end{array}$ \\
\hline $\begin{array}{l}\text { Năng lực học hỏi } \\
\text { và đẩu tư cho } \\
\text { ĐMST }\end{array}$ & 20 & 11,98 & 10,23 & 9,44 & $\begin{array}{l}\text { Cao: }>13,3 \\
\text { Trung bình: } 6,7-13,3 \\
\text { Thấp: }<6,7\end{array}$ \\
\hline
\end{tabular}




\begin{tabular}{|l|c|c|c|c|l|}
\hline & $\begin{array}{c}\text { Điểm } \\
\text { tối đa }\end{array}$ & $\begin{array}{c}\text { Chế biến } \\
\text { thực phẩm }\end{array}$ & $\begin{array}{c}\text { Dệt } \\
\text { may }\end{array}$ & $\begin{array}{c}\text { Thiết bị } \\
\text { điện-điện tử }\end{array}$ & \multicolumn{1}{|c|}{ Khoảng điểm } \\
\hline $\begin{array}{l}\text { Năng lực tạo ra } \\
\text { kết quă ĐMST }\end{array}$ & 20 & 11,01 & 11,25 & 5,95 & $\begin{array}{l}\text { Cao: }>13,3 \\
\text { Trung bình: } 6,7-13,3 \\
\text { Thấp: }<6,7\end{array}$ \\
\hline $\begin{array}{l}\text { Năng lực thương } \\
\text { mại hóa các kết } \\
\text { quả ĐMST }\end{array}$ & 30 & 17,54 & 15,81 & 10,62 & $\begin{array}{l}\text { Cao: }>20 \\
\text { Trung bình: } 10-20 \\
\text { Thấp: }<10\end{array}$ \\
\hline $\begin{array}{l}\text { Năng lực ĐMST } \\
\text { tổng hợp }\end{array}$ & 100 & 60,92 & 55,06 & 44,75 & $\begin{array}{l}\text { Cao: }>66,6 \\
\text { Trung bình: } 33,4-66,6 \\
\text { Thấp: }<33,4\end{array}$ \\
\hline
\end{tabular}

Nguồn: Tổng hợp tù kết quả khảo sát

\subsubsection{Nhận xét về phưong pháp đánh giá và các tiêu chí đo liờng}

Trong quá trình tiến hành khảo sát và phân tích dữ liệu để đánh giá NLĐMST của các DNNVV trong ba ngành chế biến thực phẩm, dệt may, và thiết bị điện-điện tử, chúng tôi thấy rằng:

Thư nhất, xét về tổng thể, đa số các doanh nghiệp không gặp khó khăn trong việc trả lời các câu hỏi dưới dạng định tính. Chỉ có khoảng $10 \%$ số doanh nghiệp không hoàn thành được bảng hỏi. Khi đi gặp doanh nghiệp để phỏng vấn sâu, các doanh nghiệp đều cho biết có thể hoàn thành được bảng hỏi.

Tuy nhiên, một số doanh nghiệp gặp khó khăn khi trả lời câu hỏi liên quan đến ĐMST đối với sản phẩm dịch vụ. Các doanh nghiệp có xu hướng nhầm lẫn giữa gói sản phẩm dịch vụ mà doanh nghiệp cung cấp cho khách hàng với các dịch vụ chăm sóc khách hàng (thuộc về hoạt động marketing, bán hàng của doanh nghiệp). Để giúp doanh nghiệp tránh nhầm lẫn, chúng tôi cho rằng trong bảng hỏi chỉ nên tập trung vào sản phẩm chính của doanh nghiệp. Nếu đó là doanh nghiệp sản xuất, chỉ nên đưa ra câu hỏi về sản phẩm hàng hóa, còn đối với doanh nghiệp dịch vụ thì chỉ đưa ra câu hỏi về gói sản phẩm dịch vụ.

Thứ hai, điểm số tính toán trong các doanh nghiệp phản ánh tương đối chính xác NLĐMST tại các doanh nghiệp mà chúng tôi tiến hành phỏng vấn sâu. Rất nhiều các doanh nghiệp trong lĩnh vực chế biến thực phâm và dệt may đều phản ánh họ học hỏi được rất nhiều từ các đối tác nước ngoài. Trong khi chỉ có số ít doanh nghiệp điện tử có cơ hội này. Các chủ doanh nghiệp khi phỏng vấn đều hào hứng với việc ĐMST nhưng cũng phản ánh các kêt quả thực sự thì không có nhiều. Việc đầu tư cho triển khai các hoạt động ĐMST đều gặp khó khăn về vốn. Đa số doanh nghiệp thường chú trọng đến cải tiến về mặt tổ chức (tìm kiếm các đối tác để hợp tác phát triển hoặc mô hình quản lý để cắt giảm chi phí). Các cải tiến về sản phẩm và marketing thường chậm thay đồi. 
Thứ $b a$, việc tổng hợp dữ liệu và tính toán điểm số từ bảng khảo sát rất dễ dàng. Với việc xây dựng các hệ số cho từng cấu phần, chỉ cần nhập các số liệu từ phiếu hỏi là chúng ta đã có được kết quả đánh giá sơ bộ về NLĐMST của doanh nghiệp theo từng cấu phần và cho toàn bộ các doanh nghiệp trong ngành. Việc tính toán dễ dàng sẽ giúp cho các cơ quan lập chính sách hoặc chuẩn đoán NLĐMST của doanh nghiệp có thể nhanh chóng sàng lọc các doanh nghiệp cần xem xét hoặc hỗ trợ.

Thứ tur, việc xác định trọng số cho các cấu phần có vẻ như là không quá quan trọng. Trong nghiên cứu này, chúng tôi đã gán trọng số điểm cao hơn cho Năng lực quản lý hoạt động ĐMST (30 điểm) và Năng lực thương mại hóa các kết quả ĐMST (30 điểm) so với Năng lực học hỏi và đầu tư cho ĐMST (20 điểm) và Năng lực tạo ra kết quả ĐMST (20 điểm). Tuy nhiên, trong tính toán mô phỏng, khi gán các trọng số ngang bằng cũng như đảo ngược cho các cấu phần thì điểm trung bình chung và từng cầu phần NLĐMST của các ngành cho các kết quả so sánh tương đối không thay đổi. Vì thế, chúng tôi cho rằng vì mục đích đánh giá nhanh, để cho đơn giản, chúng ta nên áp dụng trọng số ngang bằng cho các cấu phần.

Thứ năm, có khá nhiều doanh nghiệp không trả lời một số câu hỏi định lượng chúng tôi đưa vào trong bảng hỏi (số lượng ĐMST, số lượng dự án ĐMST bị từ bỏ). Điều này cho thấy, người trả lời có xu hướng ngại trả lời các câu hỏi có tính định lượng. Vì thế, chúng tôi cho rằng với mục tiêu đánh giá nhanh về NLĐMST của các DNNVV thì có lẽ không cần thiết phải đưa các câu hỏi định lượng vào trong bảng khảo sát.

\section{Kết luận}

Nghiên cứu này của chúng tôi nằm trong nỗ lực chung của cộng đồng nghiên cứu trong nước cũng như của các cơ quan hoạch định chính sách trong việc xây dựng bộ chỉ số đo lường NLĐMST của doanh nghiệp Việt Nam. Trước đó đã có nhiều nghiên cứu cố gắng đưa ra các cách tiểp cận riêng để đo lường mức độ ĐMST của các doanh nghiệp Việt Nam. Ở trong nghiên cứu này, chúng tôi sử dụng cách tiếp cận về đo lường ĐMST dựa trên khung khổ OSLO Manual 2005 và Chuỗi giá trị ĐMST. Bộ tiêu chí được xây dựng theo hướng phù hợp với các DNNVV của Việt Nam. Cụ thể, các bảng câu hỏi được thiết kế tương đối ngắn gọn và dễ hiểu để doanh nghiệp thực hiện. Việc tính toán, tổng hợp kết quả tương đối đơn giản, nhanh chóng để giúp cho các cơ quan quản lý hoặc doanh nghiệp có những đánh giá nhanh về NLĐMST của ngành hoặc của chính doanh nghiệp mình.

Chúng tôi kiểm định các thước đo của mình trong việc trực tiếp đo lường NLĐMST trong 3 ngành công nghiệp ưu tiên của Việt Nam: dệt may, chế biến thực phẩm và thiết bị điện-điện tử. Kết quả khảo sát thử cho thây các 
cơ quan nhà nước và doanh nghiệp có thể sử dụng bảng khảo sát và hệ thống tính điểm chúng tôi đề xuất để đánh giá nhanh NLĐMST của doanh nghiệp và của ngành. Chúng tôi cũng khuyến nghị, dựa trên quá trình khảo sát và phỏng vấn, Bảng khảo sát không nhất thiết phải đưa vào các câu hỏi định lượng. Việc tính điểm cũng không cần thiết phải gán trọng số khác nhau cho các cấu phần vì kết quả đánh giá sẽ không có sai lệch nhiều giữa các phương án gán trọng số. Ngoài ra, chúng tôi cho rằng nên có một số điều chỉnh nhỏ về ngôn từ trong Bảng khảo sát hiện tại (chủ yếu dùng cho các doanh nghiệp trong lĩnh vực chế biến) khi áp dụng cho các doanh nghiệp cung cấp dịch vụ./.

\section{TÀI LIỆU THAM KHẢO}

\section{Tiếng Việt}

1. Viện Quản lý Kinh tế Trung ương và các đối tác, 2014. Năng lục cạnh tranh và công nghệ ở cấp độ doanh nghiệp tại Việt Nam: Kết quả điều tra năm 2013". Hà Nội, Nxb Tài chính.

2. Nguyễn Việt Hòa, 2008. Nghiên cứu thực trạng hoạt động đổi mới ở doanh nghiệp công nghiẹp (trường hợp doanh nghiệp công nghiệp khu vực nhà nước). Đề tài cơ sở Viện Chiến lược và Chính sách KH\&CN.

3. Bạch Tân Sinh, 2010. Nghiên cưu hoàn thiện hệ thống chỉ số đổi mới và đánh giá thư năng lự đổi mới của doanh nghiệp ở hai ngành lựa chọn (chế tạo cơ khí và chế biến thực phẩm). Đề tài cấp Bộ, Viện Chiến lược và Chính sách KH\&CN.

4. Nguyễn Việt Hòa, 2011. Nghiên cứu phân tích và đánh giá chính sách đổi mới công nghệ cho doanh nghiệp ngành công nghiệp (Nghiên cứu doanh nghiệp ở 03 ngành công nghiệp phần mềm, dệt may và chế biến thực phẩm). Báo cáo tổng hợp đề tài cấp Bộ, Viện Chiến lược và Chính sách KH\&CN, Hà Nội.

5. Trần Ngọc Ca, Nguyễn Võ Hưng, 2012. Hướng tới một hệ thống đổi mói trong lĩnh vực nông nghiệp - truờng hợp ba sản phẩm ở Việt Nam: rau quả, chè và tôm. Hà Nội, Nxb Khoa học và kỹ thuật.

6. Phùng Xuân Nhạ, Lê Quân, 2013. Đổi mới sáng tạo của doanh nghiệp Việt Nam, ĐH Quốc gia Hà Nội.

\section{Tiếng Anh}

7. OECD, 1997. Science, technology and industry: scoreboard of indicators 1997, Paris: OECD.

8. OECD, 2005. OSLO Manual: Guidelines for collecting and interpreting innovation data.

9. OECD and Eurostat, 2005. Oslo Manual: Guidelines for collecting and interpreting innovation data, Third edition, OECD Publishing, Paris.

10. J. Schumpeter, 1934. The theory of economic development, Harvard University Press. 
11. A.Neely, R.Filippini, C.Forza, A.Vinelli, J.Hii, 2001. "A framework for analysing business performance, firm innovation and related contextual factors: perceptions of managers and policy makers in two European regions", Integrated Manufacturing Systems, 12 (2): 114-124.

12. Lawson, B. và D. Samson, 2001. "Developing innovation capability in organisations: a dynamic capabilities approach", International Journal of Innovation Management, 5 (3): $377-400$.

13. H.Romijin, M.Albaladejo, 2002. "Determinants of innovation capability in small electronics and software firms in southeast England", Research Policy, Vol. 31, pp. 1053-1067.

14. Tidd, Bessant, Pavit, 2005. Managing Innovation: Integrating Technological, Market and Organizational Change, 3rd Edition.

15. Hansen, M. and Birkinshaw, J. M., 2007. "The innovation value chain", Harvard Business Review, vol. 85 (6).

16. Arundel, A., 2007. "Innovation survey indicators: What impact on innovation policy?", Science, technology, and innovation indicators in a changing world: Responding to a policy needs, OECD.

17. Gamal, D., 2011. "How to measure organization innovativeness? An overview of innovation measurement frameworks and innovation audit/management tools", Technology Innovation and Entrepreneurship Center, truy cập ngày 10/7/2017 tại $<$ http://www.tiec.gov.eg/backend/Reports/MeasuringOrganizationInnovativeness.pdf $>$.

18. Nilsson,S., J. Wallin, A. Benaim, M.C. Annosi và R.B. Svensson, 2012. "Re-thinking innovation measurement to manage innovation-related dichotomies in practice", CINet Conference, Rome, Italy.

19. Vuong Quan Hoang, Nancy K. Napier, Vu Kim Hanh, Nguyen Manh Cuong, Tran Tri Dung, 2014. "Measuring Corporate Innovation Capacity: Experience and Implications from i2Metrix Implementation in Vietnam", ASEAN Journal of Management \& Innovation, Jan.-May 2014.

20. European Commission, 2017b. "European innovation scoreboard 2017", truy cập ngày 23/012018 tại <https://www.rvo.nl/sites/default/files/2017/06/European_ Innovation_Scoreboard_2017.pdf.>

21. Eurostat, 2017. "Innovation statistics", truy cập ngày 20/01/2018 tại $<$ http://ec.europa.eu/eurostat/statistics-explained/index.php/Innovation_statistics\# Largest_market_and_innovation.> 